\title{
Comparison of Malated Ringer's with Two Other Balanced Crystalloid Solutions in Resuscitation of Both Severe and Moderate Hemorrhagic Shock in Rats
}

\author{
Judith Keitel, ${ }^{1}$ Bjoern Hussmann, ${ }^{1}$ Sven Lendemans, ${ }^{1}$ \\ Herbert de Groot, ${ }^{2}$ and Ricarda Rohrig ${ }^{2}$ \\ ${ }^{1}$ Department for Trauma Surgery and Orthopaedics, University Hospital Essen, 45147 Essen, Germany \\ ${ }^{2}$ Institute of Physiological Chemistry, University Hospital Essen, Germany \\ Correspondence should be addressed to Ricarda Rohrig; ricarda.rohrig@uk-essen.de
}

Received 17 December 2014; Revised 11 May 2015; Accepted 12 May 2015

Academic Editor: Hartmut Jaeschke

Copyright (C) 2015 Judith Keitel et al. This is an open access article distributed under the Creative Commons Attribution License, which permits unrestricted use, distribution, and reproduction in any medium, provided the original work is properly cited.

In preclinical treatment of polytraumatized patients crystalloids are preferentially used. To avoid metabolic acidosis, metabolizable anions like lactate or acetate are used to replace chloride in these solutions. We here studied the effects of malated Ringer's in resuscitation of both shock severities in comparison to lactated and acetated Ringer's. Male Wistar rats underwent severe (mean arterial blood pressure (MAP) of $25-30 \mathrm{mmHg}$ ) or moderate (MAP $40-45 \mathrm{mmHg}$ ) hemorrhagic shock. Adjacent to the shock period animals were resuscitated with acetated (AR), lactated (LR), or malated Ringer's (MR) and observed for 150 min. MR improved survival compared with LR and AR in severe hemorrhagic shock whereas it was equally effective to LR and superior to AR in moderate hemorrhagic shock. In all other parameters tested, MR was also effective similar to the other solutions under these conditions. We conclude that MR is preferable to AR and LR in resuscitation of hemorrhagic shock independent of shock depth. The positive effects of MR may stem from the absence of any adverse impact on energy metabolism under both conditions.

\section{Introduction}

Beside craniocerebral injury, hemorrhagic shock is one of the main causes of death among trauma patients $[1,2]$. Although the resuscitation regimes changed over the past decades, the first priority is still the control of the bleeding source and the immediate restoration of the lost blood volume. In initial preclinical treatment of polytraumatized patients, crystalloids are preferentially used. Because hemorrhagic shock is strongly correlated with the occurrence of metabolic acidosis, the infusion of high amounts of chloride is considered to be counter-indicated [3]. Thus, in the resuscitation fluids chloride is partially substituted by metabolizable anions such as lactate, acetate, or malate.

During the metabolism of lactate, acetate, and malate, $\mathrm{H}^{+}$ ions are consumed (or $\mathrm{HCO}_{3}{ }^{-}$ions generated) supporting a normalization of blood $\mathrm{pH}$. Furthermore, these anions may improve energy supply to vital organs such as the heart.
For lactate, the alkalizing effect was first shown by Hartmann and later confirmed by several other groups [3-5]. We could recently demonstrate that lactate infusion may be detrimental when used in resuscitation of a severe hemorrhagic shock in rats whereas it even may prolong survival when administered in a more moderate form of hemorrhagic shock $[6,7]$.

In order to replace lactate, acetate can be used as metabolizable anion [8]. It is ubiquitously metabolized and there are no negative side effects known for the application of acetate in resuscitation solutions in severe hemorrhagic shock $[9,10]$. However, some isotonic crystalloid solutions used in hemodialysis containing acetate are assumed to mediate vasodilatory actions [11]. Although the presumption about vasodilation could not be confirmed in severe hemorrhagic shock by our group [10], there are still some efforts to find a resuscitation solution with more protective properties. Malate seems to be a promising candidate not only in treatment of metabolic acidosis but also in the maintenance 
of energy supply during hypoxic conditions [12-14]. Like acetate, it is ubiquitously metabolized and holds a pivotal role in intermediary metabolism, that is, in the tricarboxylic acid (TCA) cycle and the malate-aspartate-shuttle. However, the information about the effects of malate in direct comparison to lactate and acetate especially in the resuscitation of severe hemorrhagic shock is scarce. There is only one study comparing malated Ringer's with lactated Ringer's and normal saline in a moderate form of hemorrhagic shock [15]. Therefore, we here study the effects of lactated Ringer's (LR), acetated Ringer's (AR), and malated Ringer's (MR) in the resuscitation from a severe $(25-30 \mathrm{mmHg})$ and a moderate form (40-45 $\mathrm{mmHg}$ ) of hemorrhagic shock on survival, acidbase status, and other parameters in rats.

\section{Methods}

2.1. Chemicals/Materials. Normal saline and AR were from B. Braun (Melsungen, Germany) and MR, Ringer's solution (RS), and LR (only containing l-lactate) from Fresenius (Bad Homburg, Germany). Ketamine 10\% was from Ceva (Düsseldorf, Germany), lidocaine (Xylocaine 1\%) from AstraZeneca (Wedel, Germany), and acid citrate dextrose A solution from Baxter (Deerfield, IL). Portex catheters (inner diameter: $0.58 \mathrm{~mm}$, outer diameter: $0.96 \mathrm{~mm}$; Smiths Medical International, Hythe, UK) and medical oxygen (Air Liquide, Düsseldorf, Germany) were obtained from the vendors listed.

2.2. Animals. Male Wistar rats (400-500 g) were obtained from the central animal unit of the Essen University Hospital. Animals were kept under standardized conditions of temperature $\left(22^{\circ} \mathrm{C} \pm 1^{\circ} \mathrm{C}\right)$, humidity $(55 \% \pm 5 \%)$, and $12 \mathrm{~h} / 12 \mathrm{~h}$ lightdark cycles. The rats had free access to water, were fed with standard chow (Ssniff-Spezialditäten, Soest, Germany), and were not fasted before the experiments. All animals received human care according the standards of Annex III of the directive 2010/63/EU of the European Parliament and of the Council of 22 September 2010 on the protection of animals used for scientific purposes [16]. The experimental protocol has been approved by the North Rhine-Westfalia State Office for Nature, Environment and Consumer Protection, Recklinghausen, Germany (Landesamt für Natur, Umwelt und Verbraucherschutz Nordrhein Westfalen, Recklinghausen, Deutschland), based on the local animal protection act.

2.3. Anesthesia, Analgesia, and Surgical Procedures. Anesthesia, analgesia, catheter insertions, shock induction, resuscitation schedule, and blood sampling, were basically performed as described previously with slight modifications [10]. Rats were anesthetized with isoflurane $\left(2 \%\right.$ in $100 \%$ medical $\mathrm{O}_{2}$ at $4 \mathrm{~L} / \mathrm{min}$ for induction of anesthesia, $1 \%-1.5 \%$ at $2 \mathrm{~L} / \mathrm{min}$ throughout the experiment) through face masks connected to a vaporizer (Isofluran Vet. med. Vapor; Dräger, Lübeck, Germany) and received ketamine (50 mg/kg, s.c.) into the right chest wall for analgesia. Lidocaine $(5 \mathrm{mg} / \mathrm{kg}$, s.c.) was administered before a skin-deep incision along the right groin. Subsequently, a Portex catheter was placed within the femoral artery and the femoral vein. Each catheter was fixed with surgical suture.
2.4. Induction of Hemorrhagic Shock and Resuscitation Regimen. After insertion of the catheter, animals were allowed to adapt for $20 \mathrm{~min}$ before hemorrhagic shock was induced by removing 1-2 $\mathrm{mL}$ blood every $3 \mathrm{~min}$ through the femoral artery catheter using 2-mL syringes (Terumo, Leuven, Belgium). Bleeding was continued until the mean arterial blood pressure (MAP) dropped either to $25-30 \mathrm{mmHg}$ for severe shock or to $40-45 \mathrm{mmHg}$ for moderate shock, which typically took about $20 \mathrm{~min}$ in both groups. During the following $10 \mathrm{~min}$, the MAP was fine-tuned by sampling of smaller blood volumes $(0.5-1 \mathrm{~mL})$. For the next $60 \mathrm{~min}$, the MAP was kept between $25-30 \mathrm{mmHg}$ and $40-45 \mathrm{mmHg}$, respectively, typically without the need of any further intervention. In some individual cases small blood samples had to be withdrawn or administered to keep the MAP in the desired range. The blood used for adjustment was collected during shock induction in syringes containing acid dextrose A solution (ACDA). After the shock phase, study group-specific resuscitation fluids were infused into the femoral vein within 30 min using a syringe pump (Perfusor-Secura FT; B. Braun, Melsungen, Germany). Experiments were continued for another $150 \mathrm{~min}$, unless the animals died earlier. To compensate for fluid loss over surgical areas and the respiratory epithelium, RS $(5 \mathrm{~mL} / \mathrm{kg} / \mathrm{h})$ was infused through the femoral vein catheter throughout the experiment.

2.5. Experimental Groups. All 46 animals were randomly assigned to the following groups:

(i) sham group (no shock, no resuscitation, six animals),

(ii) moderate shock/AR group (shock, resuscitation with acetated Ringer's equal to three times the shed blood volume, eight animals),

(iii) moderate shock/LR group (shock, resuscitation with lactated Ringer's equal to three times the shed blood volume, eight animals),

(iv) moderate shock/MR group (shock, resuscitation with malated Ringer's equal to three times the shed blood volume, eight animals),

(v) severe shock/AR group (shock, resuscitation with acetated Ringer's equal to three times the shed blood volume, eight animals),

(vi) severe shock/LR group (shock, resuscitation with lactated Ringer's equal to three times the shed blood volume, eight animals),

(vii) severe shock/MR group (shock, resuscitation with malated Ringer's equal to three times the shed blood volume, eight animals).

The volume of fluid to be used for resuscitation is based on the well established $3: 1$ rule [17] taking into consideration the short intravasal half-life of crystalloid solutions.

2.6. Biomonitoring. Systolic blood pressure, diastolic blood pressure, and MAP were recorded continuously via the femoral artery catheter that was connected to a pressure transducer and displayed on a monitor. Heart rates were 
determined from systolic blood pressure spikes. The core body temperature of the rats was kept at $37.5 \pm 0.5^{\circ} \mathrm{C}$ and continuously monitored via a rectal sensor. Cooling of the animals was prevented by a cover with aluminum foil and by means of an underlying heated operating table. The oxygen saturation was recorded continuously using a pulse oximeter (OxiCliq A; Nellcor, Boulder, CO, USA) placed at the left hind limb. The breathing rate was determined based on the ventilation movements in $15 \mathrm{sec}$. All biomonitoring parameters were recorded in $10 \mathrm{~min}$ intervals.

2.7. Assessment of Blood and Plasma Parameters. Using a 2$\mathrm{mL}$ syringes containing $80 \mathrm{IU}$ electrolyte-balanced heparin (Pico50; Radiometer Medical, Brønshøj, Denmark), blood samples $(0.5-0.7 \mathrm{~mL})$ for blood gas analysis and the assessment of marker enzymes activities were taken from the femoral artery immediately after its insertion $(T=0 \mathrm{~min})$, before shock induction ( $T=30 \mathrm{~min}$ ), after the end of shock induction $(T=60 \mathrm{~min})$, immediately before the beginning of resuscitation ( $T=120 \mathrm{~min}$ ), at the end of resuscitation ( $T$ $=150 \mathrm{~min}$ ), and during the final observation phase (at $T=$ 180,240 , and $300 \mathrm{~min}$ ). For each blood sampling, animals were substituted with a $0.5 \mathrm{~mL}$ bolus of RS via the femoral artery to keep the catheter functional. Arterial blood $\mathrm{pH}$, oxygen, and carbon dioxide partial pressure $\left(\mathrm{pO}_{2}, \mathrm{pCO}_{2}\right)$, oxygen saturation, base excess (BE), hematocrit, electrolytes $\left(\mathrm{Na}^{+}, \mathrm{K}^{+}, \mathrm{Cl}^{-}, \mathrm{Ca}^{2+}\right)$, osmolarity, and lactate and glucose concentration were assessed with a blood gas analyzer (ABL 715; Radiometer, Copenhagen, Denmark). Blood plasma was obtained by centrifugation $\left(3,000 \times \mathrm{g}\right.$ for $15 \mathrm{~min}$ at $\left.25^{\circ} \mathrm{C}\right)$ and stored at $4^{\circ} \mathrm{C}$ until its use (within $4 \mathrm{~h}$ ). Plasma activities of lactate dehydrogenase (LDH) as a general marker for cell injury, creatine kinase (CK) as a marker for muscle cell injury, and glutamate-pyruvate transaminase (GPT) and glutamateoxaloacetate transaminase (GOT) as markers of liver injury were determined with a fully automated clinical chemistry analyzer (Vitalab Selectra E; VWR International, Darmstadt, Germany).

2.8. Statistical Analysis. Experiments were performed with eight animals per experimental group, except for the sham group, which consisted of six animals. Data are expressed as mean values \pm SEM. Outliers were removed after boxplot analysis. Comparisons among multiple groups were performed using one-way analysis of variance (ANOVA) either for nonrecurring or for repeated measures followed by Fisher (LSD, least significant difference) post hoc analysis. Survival curves were generated according to the KaplanMeier method and were compared with the log-rank test. $P<0.05$ was considered significant.

\section{Results}

3.1. Survival. All animals of the sham group survived the experiment (data not shown). In the severe shock groups, the animals receiving LR were the first who died (beginning at $T=160 \mathrm{~min}$; the last one died $30 \mathrm{~min}$ before the end of the experiment at $T=270 \mathrm{~min}$; Figure 1). Although one animal of

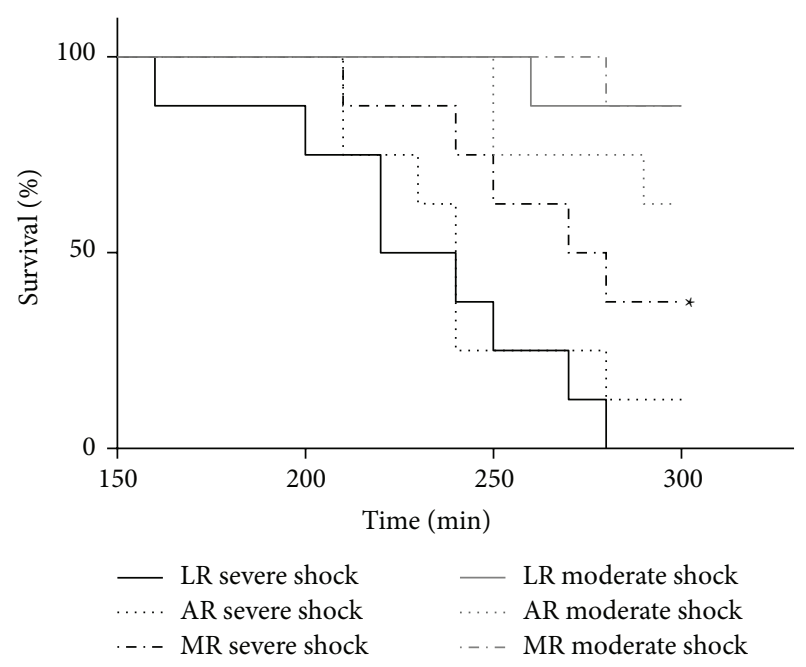

FIGURE 1: Effects of lactated, acetated, and malated Ringer's on survival in severely/moderately shocked rats. Rats underwent severe and moderate hemorrhage and then were resuscitated with either lactated Ringer's (LR), acetated Ringer's (AR), or malated Ringer's solution (MR). The survival per group is shown as Kaplan-Meier plot ( $n=7-8$ animals; log-rank ${ }^{*} P<0.05$ (versus severe shock/AR and LR)). Note: the time point at which the first animal among the shock groups died (e.g., $T=160 \mathrm{~min}$ for the severe shock/LR group) was defined as the last one where measurements between the respective groups were compared.

the severe shock/AR group survived the whole experimental time, the median survival of the shock/LR and the shock/AR group was similar (230 versus $240 \mathrm{~min}$ ). In marked contrast, resuscitation with MR significantly prolonged survival (first rat died at $T=210 \mathrm{~min}$, median survival $275 \mathrm{~min}$ ) compared to LR. Three animals of this group survived until the end of the experiment.

In the moderate shock/MR and LR group, only one animal died during the experiment (at $T=280 \mathrm{~min}$ and $T=$ $260 \mathrm{~min}$, resp.). Three of the moderate shock/AR group rats died before the end of the experiment (two animals at $T=$ $250 \mathrm{~min}$ and one at $T=290 \mathrm{~min}$ ).

3.2. Mean Arterial Blood Pressure. In the sham group animals the mean arterial blood pressure (MAP) remained fairly constant at $100 \mathrm{mmHg}$ throughout the experiment (data not shown). In the animals of the shock groups, the MAP was decreased either to $25-30 \mathrm{mmHg}$ (severe shock) or to $40-$ $45 \mathrm{mmHg}$ (moderate shock) within $30 \mathrm{~min}$ and subsequently maintained in these ranges for $60 \mathrm{~min}$ (Figure 2). In the severe shock groups, upon resuscitation, the MAP increased to values around $70 \mathrm{mmHg}$; subsequently, in the postresuscitation phase it rapidly decreased to about $40-45 \mathrm{mmHg}$ without significant differences between the groups. Upon resuscitation, the MAP of the moderate shock/MR group recovered to about $110 \mathrm{mmHg}$ whereas it increased only to about 95 and $85 \mathrm{mmHg}$ in the moderate shock/LR and AR group, respectively. In all moderate shock groups, the MAP 


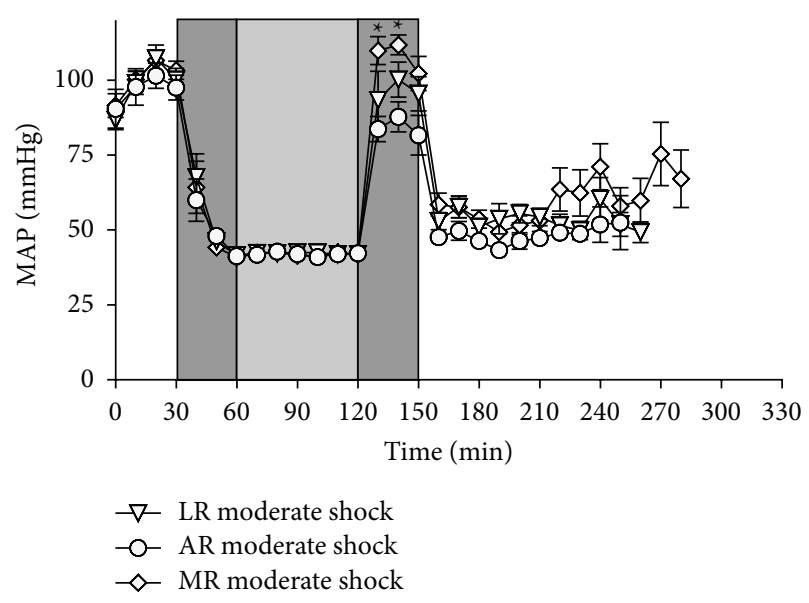

(a)

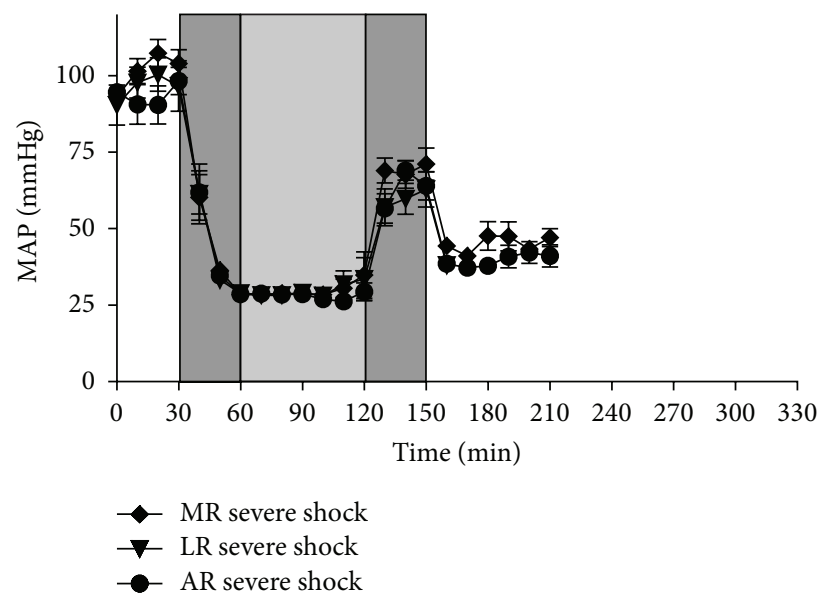

(b)

FIGURE 2: Effects of lactated, acetated, and malated Ringer's on MAP in severely/moderately shocked rats. Rats underwent moderate and severe hemorrhage (shock induction: dark grey; shock phase: light grey) and then were resuscitated (dark grey) with either lactated Ringer's (LR), acetated Ringer's (AR), or malated Ringer's solution (MR) and observed for further 150 min or until the first animal of the respective group died. (a) Mean arterial blood pressure (MAP) in moderate hemorrhagic shock of LR, AR, and MR and (b) MAP in severe hemorrhagic shock of LR, AR, and MR treated animals. Shown are mean values \pm SEM $(n=8$ animals per shock group; $P<0.05$ (versus moderate shock/AR and LR)). SEM values not visible are hidden by the symbols.

declined to around $50-65 \mathrm{mmHg}$ in the postresuscitation phase.

3.3. Hematocrit and Plasma Electrolyte Concentrations. In the sham group animals, no significant changes in hematocrit and plasma electrolyte concentrations were observed (data not shown). In all shock groups, independent of shock depth, there were no alterations in the $\mathrm{Na}^{+}$and $\mathrm{Ca}^{2+}$ concentrations, but slight elevations in the $\mathrm{Cl}^{-}$and $\mathrm{K}^{+}$concentrations during the postresuscitation phase. In contrast to the moderate shock groups where the hematocrit remained stable during the postresuscitation phase, in the severe shock groups it slightly increased after resuscitation.

3.4. Acid-Base Status. The $\mathrm{pH}, \mathrm{BE}$, and $\mathrm{pCO}_{2}$ of the sham group were stable at values of $7.35,-3 \mathrm{mmol} / \mathrm{L}$, and $45 \mathrm{mmHg}$, respectively, throughout the experiment (data not shown). During shock induction, the shock and the resuscitation phase, the $\mathrm{pH}$ decreased to values around 7.1 in the severe shock group animals, but remained around 7.35 in the moderate shock groups (Figure 3(a)). In the postresuscitation phase, the $\mathrm{pH}$ increased somewhat to values around 7.3 in the severe shock groups and to around 7.4 in the moderate shock groups. During shock induction and the shock phase, the BE rapidly decreased to around $-18 \mathrm{mmol} / \mathrm{L}$ (severe shock/LR group), $-15 \mathrm{mmol} / \mathrm{L}$ (severe shock/MR and AR group), and $-6 \mathrm{mmol} / \mathrm{L}$ (moderate shock groups), respectively, and varied around these values during the resuscitation and the postresuscitation phase (Figure 3(b)). In all shock groups, the $\mathrm{pCO}_{2}$ rapidly decreased during shock induction to around $35 \mathrm{mmHg}$, remained around this value in the shock phase, regained $40 \mathrm{mmHg}$ at the end of the resuscitation phase, but subsequently decreased to about $30 \mathrm{mmHg}$ (Figure 3(c)). The $\mathrm{pO}_{2}$ varied around $400 \mathrm{mmHg}$ in all groups studied during the whole experiment (Figure 3(d)).

3.5. Lactate and Glucose Concentrations. Sham group plasma lactate and glucose concentration remained stable at around $1 \mathrm{mmol} / \mathrm{L}$ and $200 \mathrm{mg} / \mathrm{dL}$, respectively, during the whole experiment (data not shown). In the severe shock groups, the plasma lactate concentration increased to about $8 \mathrm{mmol} / \mathrm{L}$ during shock induction and the shock phase. During resuscitation and the postresuscitation phase it either remained at this level (severe shock/LR group) or declined to about 5 (severe shock/AR group) and $3 \mathrm{mmol} / \mathrm{L}$ (severe shock/MR group), respectively. In all moderate shock groups, the plasma lactate concentration rose to 2 to $3 \mathrm{mmol} / \mathrm{L}$ during shock induction and the shock phase but subsequently decreased to about $2 \mathrm{mmol} / \mathrm{L}$ (Figure 4(a)). The blood glucose concentration rapidly increased during shock induction in all shock groups to about 350 (severe shock) and $250 \mathrm{mg} / \mathrm{dL}$ (moderate shock; Figure 4(b)), respectively. During shock, it varied around $250 \mathrm{mg} / \mathrm{dL}$ independent of shock depth and subsequently decreased during the resuscitation and the postresuscitation phase, finally reaching values of approximately $80 \mathrm{mg} / \mathrm{dL}$ (severe shock) and $100 \mathrm{mg} / \mathrm{dL}$ (moderate shock), respectively.

3.6. Plasma Enzyme Activities. In the sham group, plasma GOT, GPT, LDH and CK activities did not exceed $80 \mathrm{U} / \mathrm{L}$, $60 \mathrm{U} / \mathrm{L}, 160 \mathrm{U} / \mathrm{L}$, and $350 \mathrm{U} / \mathrm{L}$, respectively, throughout the whole experiment (data not shown), in order to match the figure. Plasma enzyme activities began to rise upon resuscitation and in the postresuscitation phase without any differences within the groups of one shock depth (Figure 5). The activities 


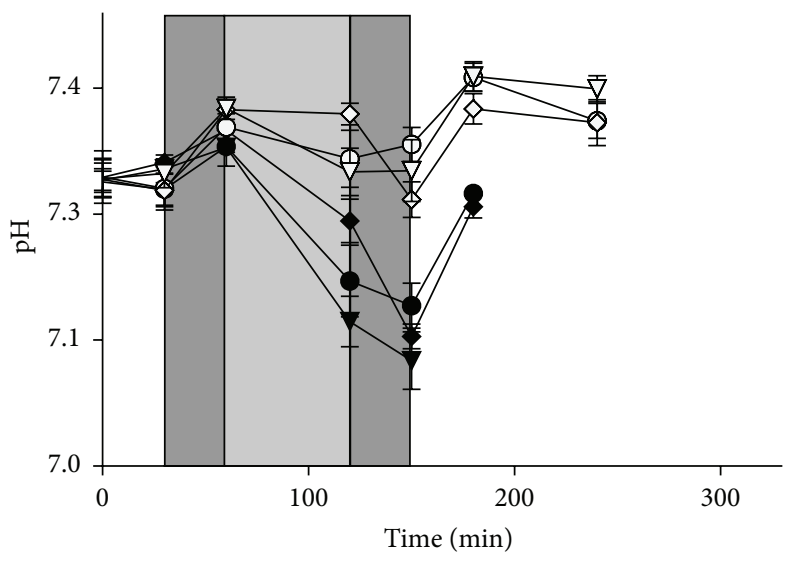

(a)

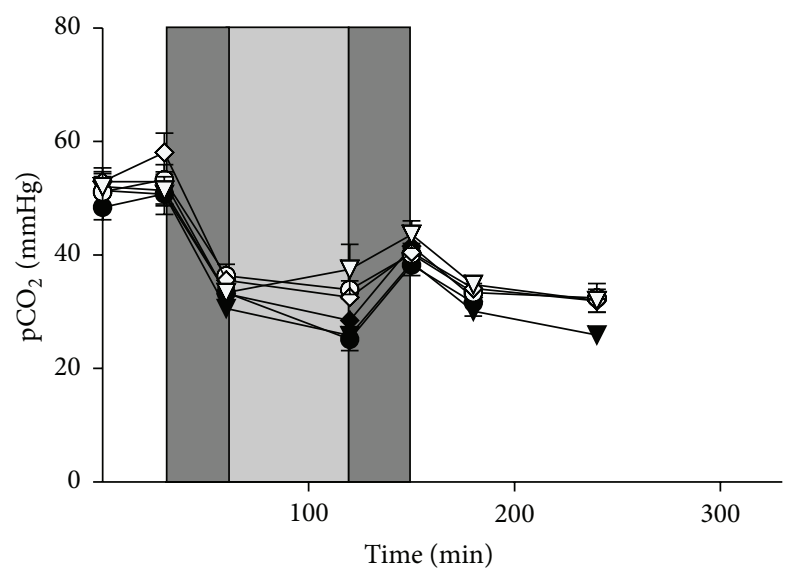

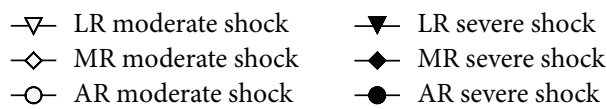

(c)

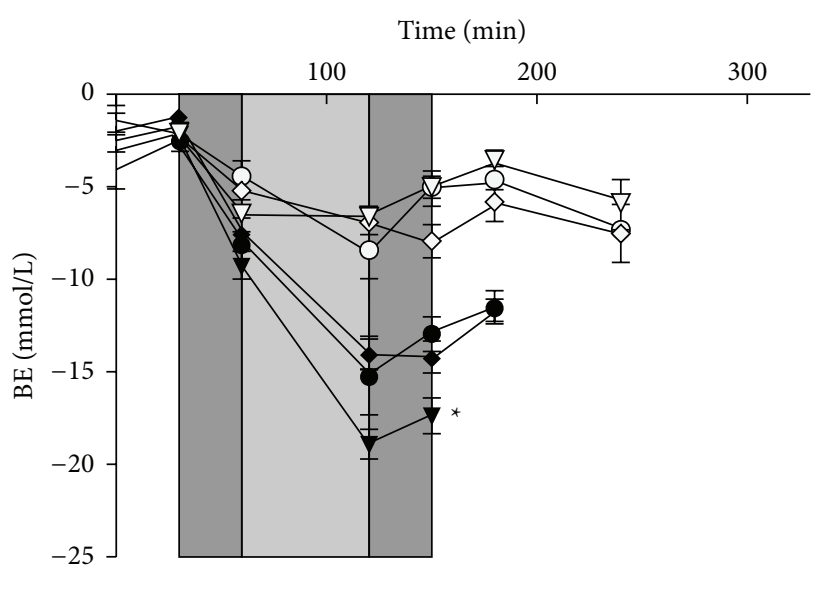

(b)

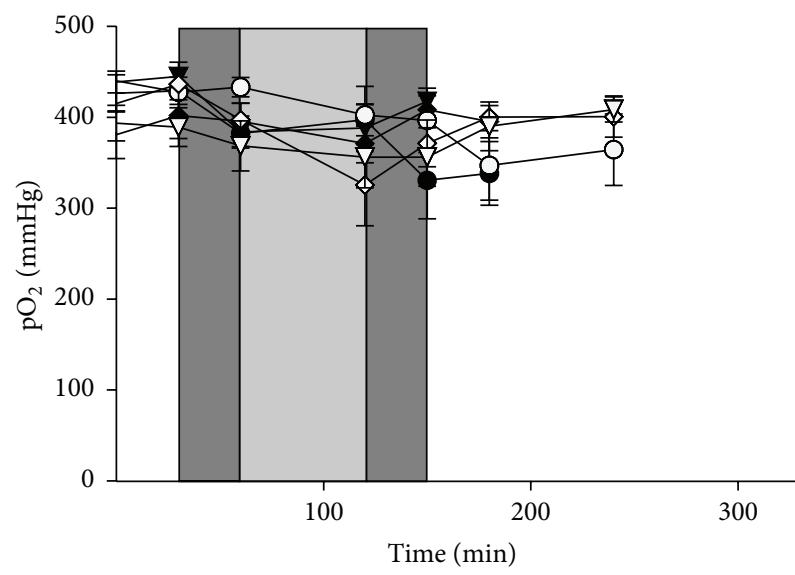

$\checkmark$ MR moderate shock $\multimap$ MR severe shock

(d)

FigurE 3: Effects of lactated, acetated, and malated Ringer's on the acid-base balance in severely/moderately shocked rats. Rats underwent severe and moderate hemorrhage (shock induction: dark grey; shock phase: light grey) and then were resuscitated (dark grey) with either lactated Ringer's (LR), acetated Ringer's (AR), or malated Ringer's solution (MR) and observed for further 150 min or until the first animal of the respective group died. (a) Blood $\mathrm{pH}$, (b) base excess, (c) $\mathrm{CO}_{2}$ partial pressure $\left(\mathrm{pCO}_{2}\right.$ ), and (d) $\mathrm{O}_{2}$ partial pressure (pO $\mathrm{p}_{2}$ ). Values are means \pm SEM $\left(n=8\right.$ animals per shock group; ${ }^{*} P<0.05$ (versus severe shock/AR)). SEM values not visible are hidden by the symbols.

of the moderate shock groups, however, exhibited a delayed increase in comparison with the severe shock groups.

\section{Discussion}

At present, the most frequently applied metabolizable anion in crystalloid solutions is lactate. LR was shown to be effective in the treatment of metabolic disorders in children [4] and it provided an improved cardiac performance and ameliorated outcome in the treatment of hemorrhagic shock when compared with normal saline [3, 18, 19]. In our hands, however, LR was superior to pure Ringer's solution in the resuscitation of a moderate form of hemorrhagic shock, but exhibited clearly detrimental effects in resuscitation of severe hemorrhagic shock $[6,7,10]$. Similar results were obtained here. In severe hemorrhagic shock, the shortest survival time was found in those animals resuscitated with LR (Figure 1), while in moderate hemorrhagic shock resuscitation with LR even slightly improved survival and to some extent increased MAP (Figure 2) as compared with AR. This opposing behaviour of LR upon resuscitation following severe or moderate hemorrhagic shock may be best explained by differences in lactate catabolism under both conditions. Following severe hemorrhagic shock, administration of LR provides an additional lactate burden (increase in lactate concentration; see also Figure 4), which may result in an inhibition of glycolysis and thus energy production [7, 2022]. In contrast, after moderate hemorrhagic shock lactate 


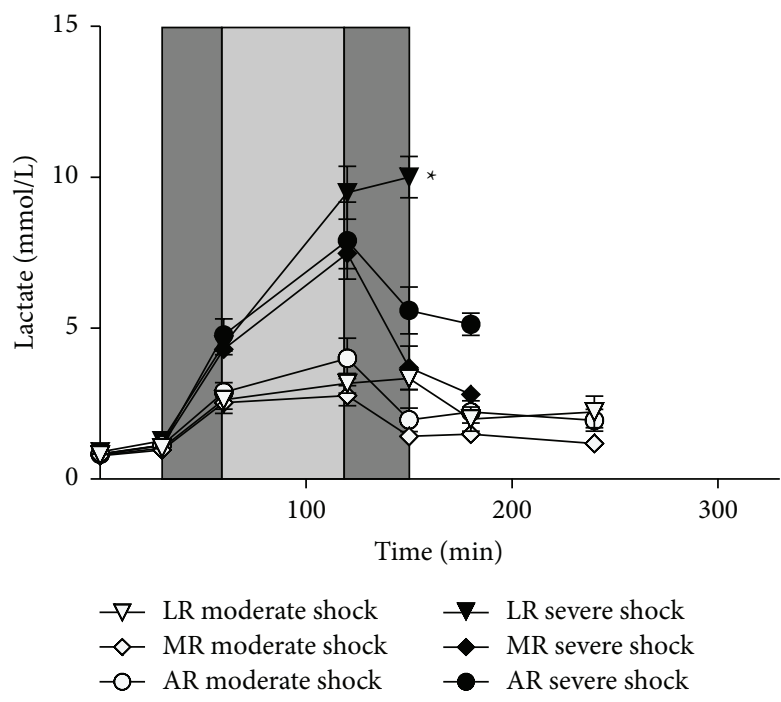

(a)

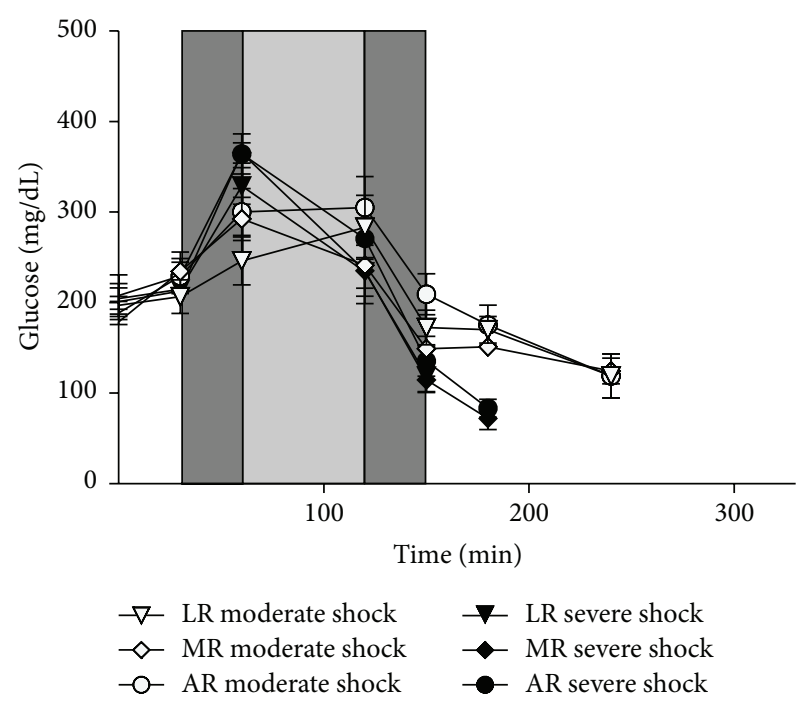

(b)

FIGURE 4: Effects of lactated, acetated, and malated Ringer's on lactate and glucose concentration in severely/moderately shocked rats. Rats underwent severe and moderate hemorrhage (shock induction: dark grey; shock phase: light grey) and then were resuscitated (dark grey) with either lactated Ringer's (LR), acetated Ringer's (AR), or malated Ringer's solution (MR) and observed for further 150 min or until the first animal of the respective group died. Blood glucose (a) and lactate concentrations (b). Values are means \pm SEM ( $n=8$ animals per shock group; ${ }^{*} P<0.05$ (versus severe shock/LR)). SEM values not visible are hidden by the symbols.

is readily metabolized, as also indicated by the improved blood $\mathrm{pH}$ under these conditions (Figure 3), and thus may especially ameliorate cardiac function $[6,19]$.

For acetate supplied in saline solutions, mainly beneficial effects have been described, such as a positive influence on the acid-base balance in and after hemorrhagic shock, presumably resulting from its fast and ubiquitous catabolic degradation [23-25]. We recently showed that AR was superior to LR in severe hemorrhagic shock in rats [10]. In line with these results, in the present study in severe hemorrhagic shock AR tended to prolong survival and clearly improved acid-base status as compared to LR (Figures 3 and 4). In moderate hemorrhagic shock, however, AR appears to shorten survival as compared with both other solutions (Figure 1) and resuscitation with $\mathrm{AR}$ resulted in a smaller increase in MAP as compared with LR and especially with MR (Figure 2). An explanation for this unexpected behavior might be that acetate is a less appropriate substrate, especially of the heart, as compared to malate and lactate under these conditions.

Although the metabolism of malate and its effects on metabolic acidosis and the cardiovascular system under hypoxic conditions have been extensively studied [12, 13, 2628 ], there are only a few studies dealing with its efficacy in the treatment of hemorrhagic shock $[14,15]$. In the study of Dai et al., where a moderate form of hemorrhagic shock was applied $(40 \mathrm{mmHg}$, as in the moderate hemorrhagic shock of the present experiments), remarkable improvements in MAP, improved acid-base status, and reduced organ injury were shown in male Sprague-Dawley rats treated with a self-established Ringer's malate solution as compared with
Ringer's lactate and normal saline [15]. In the present study, comparable results were obtained in moderate hemorrhagic shock. Even more impressive, however, resuscitation with MR led to an increased survival in severely shocked animals in relation to both LR and AR (Figure 1). Furthermore, MR somewhat improved acid-base balance as compared to LR under these conditions (Figure 3). This superiority of $\mathrm{MR}$ is most likely related to malate's pivotal role in the intermediary metabolism. Under aerobic conditions, malate is readily oxidized to oxaloacetate via the tricarboxylic acid cycle thereby exerting anaplerotic functions to fuel ATP synthesis [29]. It is also part of a shuttling system in the inner mitochondrial membrane, the malate-aspartate shuttle, ensuring the transport of reduction equivalents generated in the cytosol into the mitochondrion [30, 31]. It is well known that the malate-aspartate shuttle decreases cytosolic $\mathrm{NADH} / \mathrm{NAD}^{+}$ratio and thus may support oxidative phosphorylation and glycolysis, a mechanism which may even work under hypoxic (but not anoxic) conditions [12, 32, 33]. Moreover, in hypoxia malate may stimulate substratelevel phosphorylation by partly inverted reactions of the tricarboxylic acid cycle [34-36]. Beside its effects on energy metabolism, malate may also exert antioxidative properties due to providing reduction equivalents for the elimination of reactive oxygen species [15].

\section{Conclusions}

The present study indicates that the successful application of LR and AR in the treatment of hemorrhagic shock is 


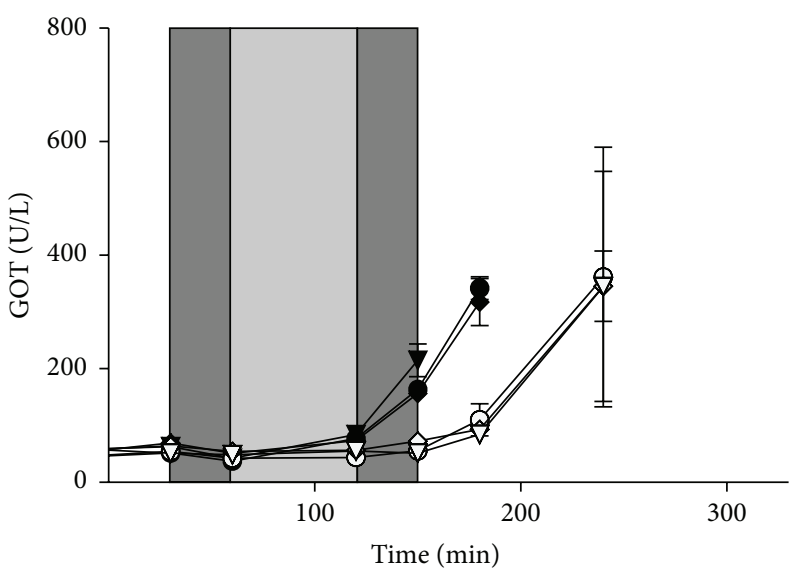

(a)

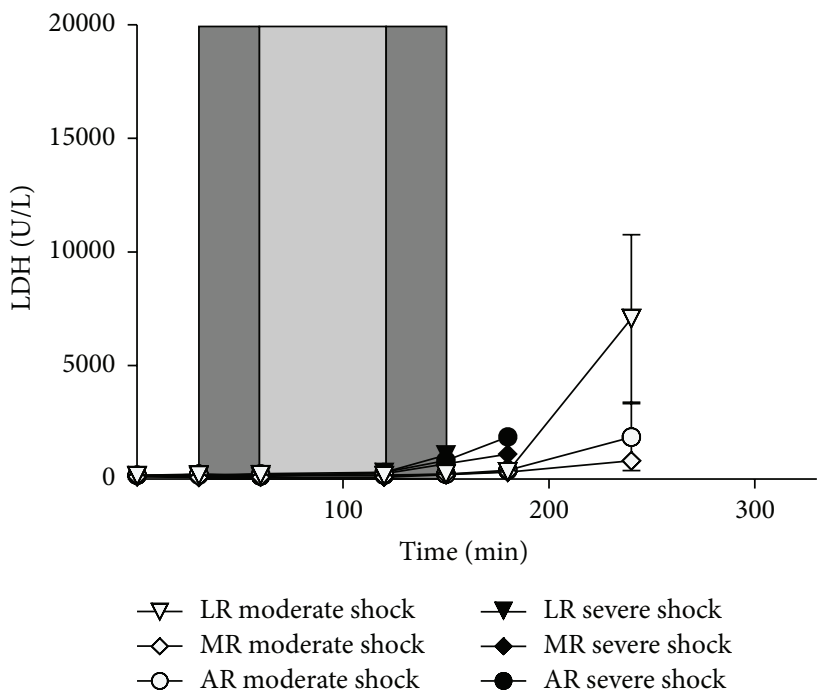

(c)

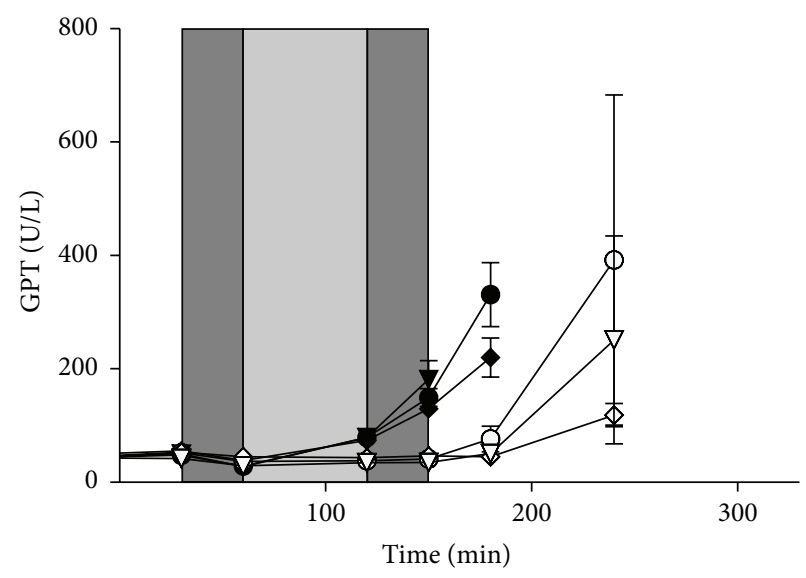

(b)

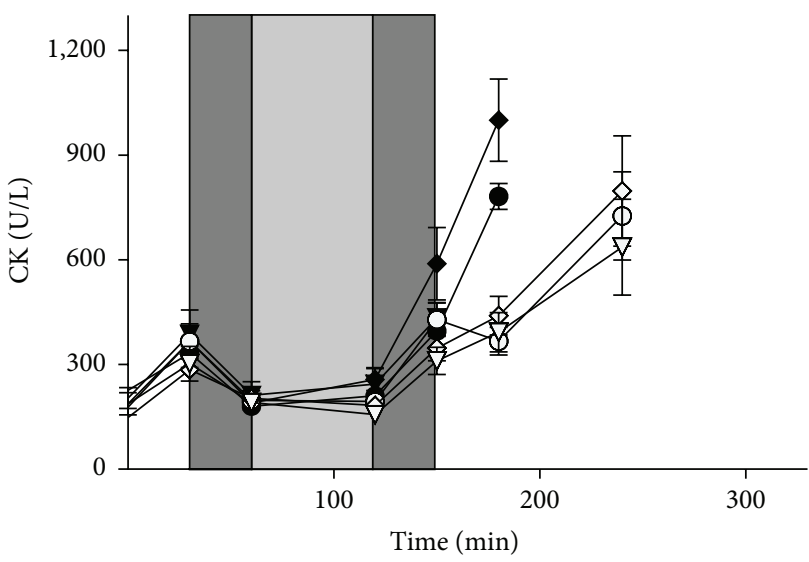

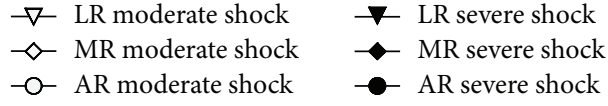

(d)

FIGURE 5: Effects of lactated, acetated, and malated Ringer's on plasma enzyme activities in severely/moderately shocked rats. Rats underwent severe and moderate hemorrhage (shock induction: dark grey; shock phase: light grey) and then were resuscitated (dark grey) with either lactated Ringer's (LR), acetated Ringer's (AR), or malated Ringer's solution (MR) and observed for further 150 min or until the first animal of the respective group died. GOT activity (a), GPT activity (b), LDH activity (c), and CK activity (d). Values are means \pm SEM ( $n=8$ animals per shock group); SEM values not visible are hidden by the symbols.

dependent on shock severity. While LR provided almost optimal results in moderate hemorrhagic shock, AR seems more advantageous in severe hemorrhagic shock. Independent of shock severity, however, MR appears to be the superior resuscitation solution. In moderate hemorrhagic shock it provided results comparable to LR and in severe hemorrhagic shock outcome was clearly improved as compared to both other solutions. Thus, the use of MR, especially in preclinical resuscitation schedules in which the magnitude of shock can only be estimated, should be preferred. However, the results presented here apply only for the experimental conditions used here, that is, severe and moderate hemorrhagic shock with a controlled blood loss but without further attempts at treatment. Whether they can be adopted to the treatment of polytraumatized patients remains to be elucidated.

\section{Abbreviations}

ACD-A: Acid citrate dextrose-A

ANOVA: Analysis of variance

AR: $\quad$ Acetated Ringer's

BE: $\quad$ Base excess

bpm: Beats per min

CK: $\quad$ Creatine kinase

GOT: Glutamate-oxaloacetate transaminase

GPT: Glutamate-pyruvate transaminase

Hct: Hematocrit

LDH: $\quad$ Lactate dehydrogenase

LR: $\quad$ Lactated Ringer's

MAP: $\quad$ Mean arterial blood pressure 
MR: Malated Ringer's

$\mathrm{pCO}_{2}$ : Carbon dioxide partial pressure

$\mathrm{pO}_{2}$ : Oxygen partial pressure.

\section{Disclosure}

The address of Judith Keitel, Bjoern Hussmann, and Sven Lendemans was University Hospital Essen, Essen, Germany, when the data was collected; however, the current address is Alfried Krupp Hospital, Essen, Germany.

\section{Conflict of Interests}

The authors declare that there is no conflict of interests regarding the publication of this paper.

\section{References}

[1] P. C. Spinella and J. B. Holcomb, "Resuscitation and transfusion principles for traumatic hemorrhagic shock," Blood Reviews, vol. 23, no. 6, pp. 231-240, 2009.

[2] D. S. Kauvar and C. E. Wade, "The epidemiology and modern management of traumatic hemorrhage: US and international perspectives," Critical Care, vol. 9, supplement 1, no. 5, pp. S1-S9, 2005.

[3] S. R. Todd, D. Malinoski, P. J. Muller, and M. A. Schreiber, "Lactated Ringer's is superior to normal saline in the resuscitation of uncontrolled hemorrhagic shock," Journal of Trauma, vol. 62, no. 3, pp. 636-639, 2007.

[4] A. F. Hartmann and M. J. Senn, "Sudies in the metabolism of sodium $r$-lactate. II. Response of human subjects with acidosis to the intravenous injection of sodium $r$-lactate," The Journal of Clinical Investigation, vol. 11, pp. 337-344, 1932.

[5] K. Ikeya, S. Kashimoto, M. Kume, and T. Kumazawa, "Effects of lactated ringer solution and acetated ringer solution on hepatic ATP and L/P ratio in rats subjected to acute hemorrhage," Masui, vol. 47, no. 1, pp. 36-41, 1998.

[6] B. Hussmann, S. Lendemans, H. de Groot, and R. Rohrig, "Volume replacement with Ringer-lactate is detrimental in severe hemorrhagic shock but protective in moderate hemorrhagic shock: studies in a rat model," Critical Care, vol. 18, no. 1, article R5, 2014.

[7] R. Rohrig, T. Rönn, S. Lendemans, T. Feldkamp, H. de Groot, and F. Petrat, "Adverse effects of resuscitation with lactated ringer compared with ringer solution after severe hemorrhagic shock in rats," Shock, vol. 38, no. 2, pp. 137-145, 2012.

[8] A. McCague, M. Dermendjieva, R. Hutchinson, D. T. Wong, and N. Dao, "Sodium acetate infusion in critically ill trauma patients for hyperchloremic acidosis," Scandinavian Journal of Trauma, Resuscitation and Emergency Medicine, vol. 19, article 24, 2011.

[9] E. Almac, U. Aksu, R. Bezemer et al., "The acute effects of acetate-balanced colloid and crystalloid resuscitation on renal oxygenation in a rat model of hemorrhagic shock," Resuscitation, vol. 83, no. 9, pp. 1166-1172, 2012.

[10] R. Rohrig, C. Wegewitz, S. Lendemans, F. Petrat, and H. de Groot, "Superiority of acetate compared with lactate in a rodent model of severe hemorrhagic shock," Journal of Surgical Research, vol. 186, no. 1, pp. 338-345, 2014.

[11] P. L. Kirkendol, J. E. Pearson, J. D. Bower, and R. D. Holbert, "Myocardial depressant effects of sodium acetate," Cardiovascular Research, vol. 12, no. 2, pp. 127-136, 1978.
[12] R. J. Wiesner, P. Rosen, and M. K. Grieshaber, "Pathways of succinate formation and their contribution to improvement of cardiac function in the hypoxic rat heart," Biochemical Medicine and Metabolic Biology, vol. 40, no. 1, pp. 19-34, 1988.

[13] D. G. Penney and J. Cascarano, "Anaerobic rat heart. Effects of glucose and tricarboxylic acid-cycle metabolites on metabolism and physiological performance," Biochemical Journal, vol. 118, no. 2, pp. 221-227, 1970.

[14] H. Winkler and B. Pöthig, "Mortality in hemorrhagic shock following infusion of a sodium bicarbonate-sodium malate solution (experiments in rats)," Bruns' Beiträge für Klinische Chirurgie, vol. 216, no. 5, pp. 470-477, 1968.

[15] Z.-L. Dai, J. Wu, C. Meng, F. Zeng, Y. Yang, and S.-L. Yao, "Ringer's malate solution protects against the multiple organ injury and dysfunction caused by hemorrhagic shock in rats," Shock, vol. 38, no. 3, pp. 268-274, 2012.

[16] Directive 2010/63/EU of the European Parliament and the Council of 22 September 2010 on the protection of animals used for scientific purposes, http://eurlex.europa.eu/LexUriServ/ LexUriServ.do?uri=OJ:L:2010:276:0033:0079:en:PDF.

[17] S. A. Stern, "Low-volume fluid resuscitation for presumed hemorrhage shock: helpful or harmful?" Current Opinion in Critical Care, vol. 7, no. 6, pp. 422-430, 2001.

[18] L. N. Kiraly, J. A. Differding, T. M. Enomoto et al., "Resuscitation with normal saline (NS) vs. lactated Ringer's (LR) modulates hypercoagulability and leads to increased blood loss in an uncontrolled hemorrhagic shock swine model," The Journal of Trauma, vol. 61, pp. 57-65, 2006.

[19] J. A. Kline, L. R. Thornton, G. D. Lopaschuk, R. W. Barbee, and J. A. Watts, "Lactate improves cardiac efficiency after hemorrhagic shock," Shock, vol. 14, no. 2, pp. 215-221, 2000.

[20] T. C. Leite, R. G. Coelho, D. D. Silva, W. S. Coelho, M. M. Marinho-Carvalho, and M. Sola-Penna, "Lactate downregulates the glycolytic enzymes hexokinase and phosphofructokinase in diverse tissues from mice," FEBS Letters, vol. 585, no. 1, pp. 92-98, 2011.

[21] R. A. Robergs, F. Ghiasvand, and D. Parker, "Biochemistry of exercise-induced metabolic acidosis," The American Journal of Physiology: Regulatory Integrative and Comparative Physiology, vol. 287, no. 3, pp. R502-R516, 2004.

[22] M. J. Rovetto, W. F. Lamberton, and J. R. Neely, "Mechanisms of glycolytic inhibition in ischemic rat hearts," Circulation Research, vol. 37, no. 6, pp. 742-751, 1975.

[23] M. Kveim and R. Nesbakken, "Utilization of exogenous acetate during canine haemorrhagic shock," Scandinavian Journal of Clinical and Laboratory Investigation, vol. 39, no. 7, pp. 653-658, 1979.

[24] Y. Matsuda, T. Sakurai, M. Iino, and K. Nakayama, "Comparative study on the effects of acetated Ringer's solution, lactated Ringer's solution, Ringer's solution, and $5 \%$ glucose-acetated Ringer's solution on canine hemorrhagic shock," Journal of Anesthesia, vol. 8, pp. 326-333, 1994.

[25] M. Kveim, R. Nesbakken, and J. E. Bredesen, "Plasma acetate concentrations during canine haemorrhagic shock," Scandinavian Journal of Clinical and Laboratory Investigation, vol. 39, no. 7, pp. 645-652, 1979.

[26] C. Hohl, R. Oestreich, P. Rosen, R. Wiesner, and M. Grieshaber, "Evidence for succinate production by reduction of fumarate during hypoxia in isolated adult rat heart cells," Archives of Biochemistry and Biophysics, vol. 259, no. 2, pp. 527-535, 1987. 
[27] J. B. Brückner, "Comparative studies on the effect of sodium lactate, sodium acetate and sodium malate in metabolic acidosis," Der Anaesthesist, vol. 19, no. 6, pp. 219-223, 1970.

[28] J. M. Tager, "Synthesis of glutamate from $\alpha$-oxoglutarate and ammonia in rat-liver mitochondria: III. Malate hydrogen donor," Biochimica et Biophysica Acta, vol. 77, pp. 258-265, 1963.

[29] O. E. Owen, S. C. Kalhan, and R. W. Hanson, "The key role of anaplerosis and cataplerosis for citric acid cycle function," The Journal of Biological Chemistry, vol. 277, no. 34, pp. 3040930412, 2002.

[30] B. Safer, "The metabolic significance of the malate aspartate cycle in heart," Circulation Research, vol. 37, no. 5, pp. 527-533, 1975.

[31] W. V. V. Greenhouse and A. L. Lehninger, "Magnitude of malateaspartate reduced nicotinamide adenine dinucleotide shuttle activity in intact respiring tumor cells," Cancer Research, vol. 37, no. 11, pp. 4173-4181, 1977.

[32] L. H. Opie, "Myocardial metabolism and heart disease," Japanese Circulation Journal, vol. 42, no. 11, pp. 1223-1247, 1978.

[33] H. Safer and J. R. Williamson, "Functional significance of the malate-aspartate shuttle for the oxidation of cytoplasmic reducing equivalents in rat heart," Recent Advances in Studies on Cardiac Structure and Metabolism, vol. 1, pp. 34-43, 1972.

[34] R. J. Wiesner, A. Deussen, M. Borst, J. Schrader, and M. K. Grieshaber, "Glutamate degradation in the ischemic dog heart: contribution to anaerobic energy production," Journal of Molecular and Cellular Cardiology, vol. 21, no. 1, pp. 49-59, 1989.

[35] C. Chinopoulos, "Which way does the citric acid cycle turn during hypoxia? The critical role of $\alpha$-ketoglutarate dehydrogenase complex," Journal of Neuroscience Research, vol. 91, no. 8, pp. 1030-1043, 2013.

[36] W. L. Chick, R. Weiner, J. Cascareno, and B. W. Zweifach, "Influence of Krebs-cycle intermediates on survival in hemorrhagic shock," The American Journal of Physiology, vol. 215, no. 5, pp. 1107-1110, 1968. 


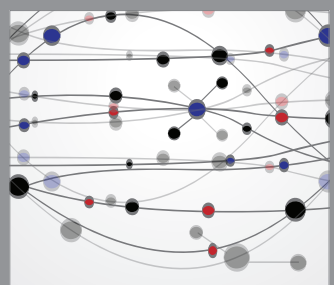

The Scientific World Journal
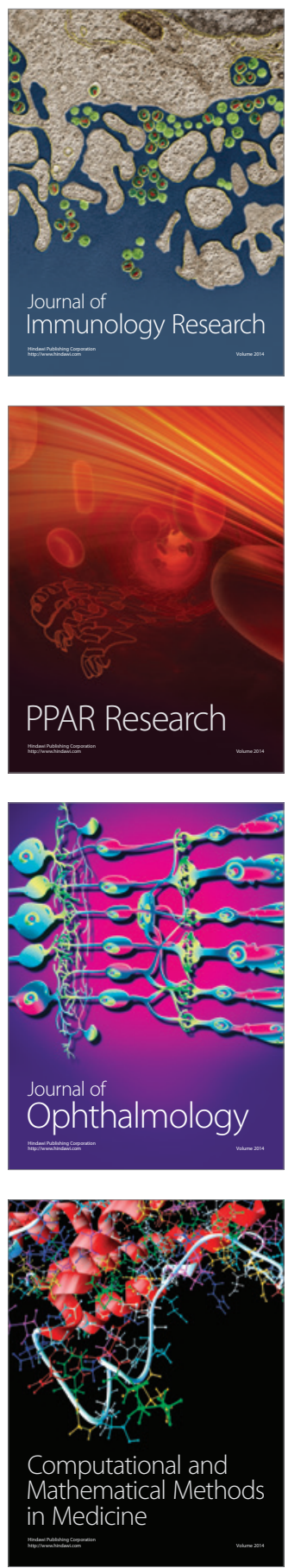

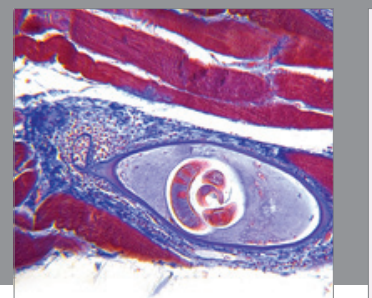

Gastroenterology

Research and Practice
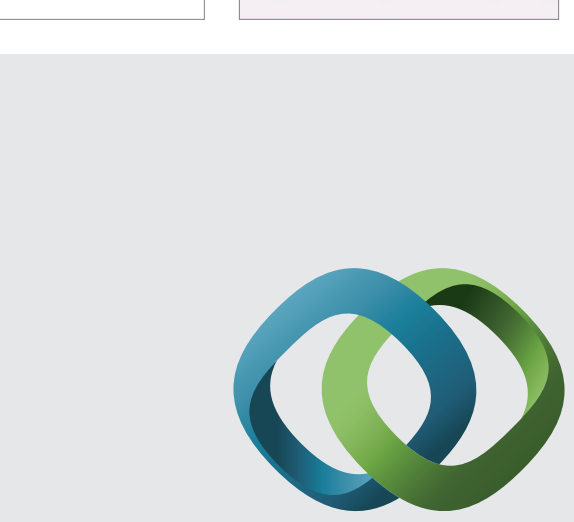

\section{Hindawi}

Submit your manuscripts at

http://www.hindawi.com
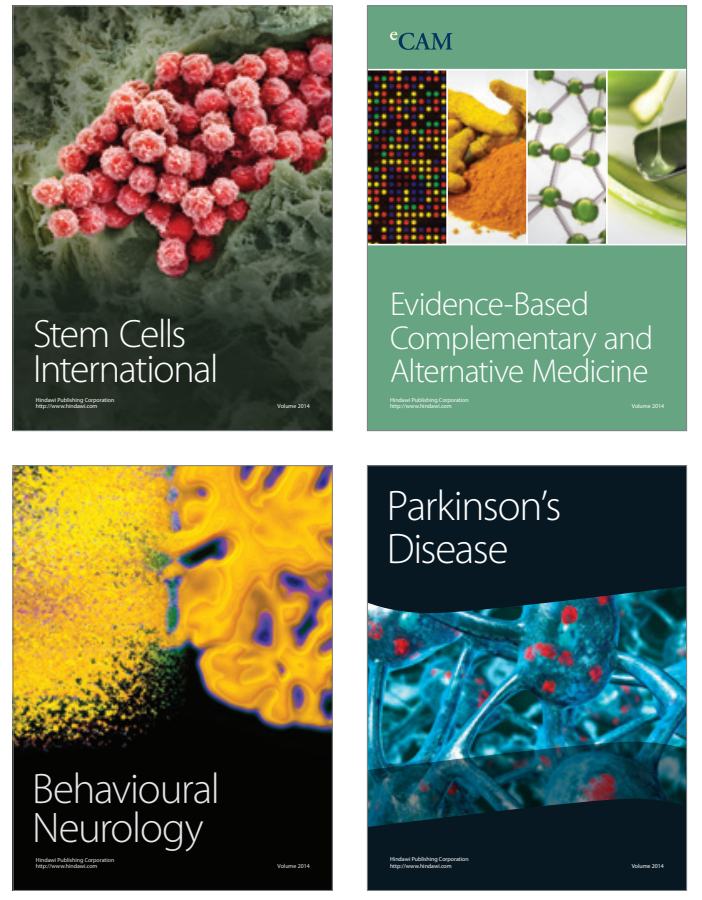
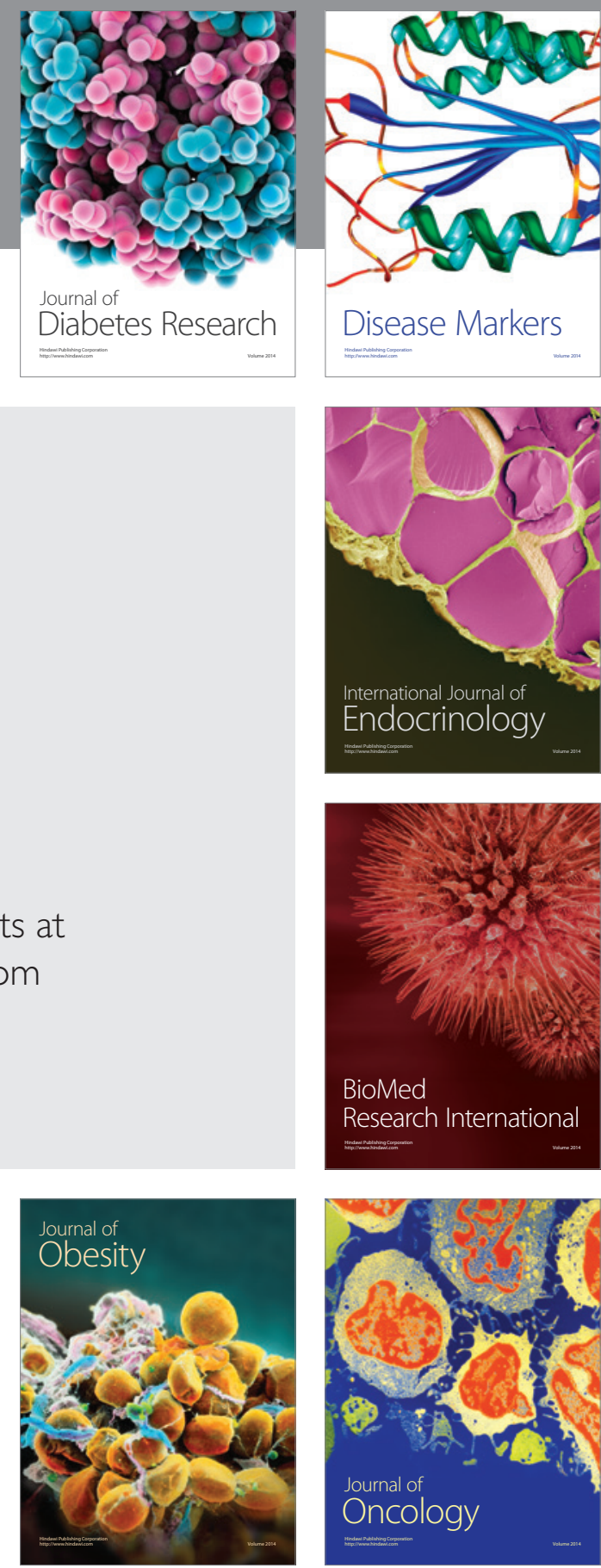

Disease Markers
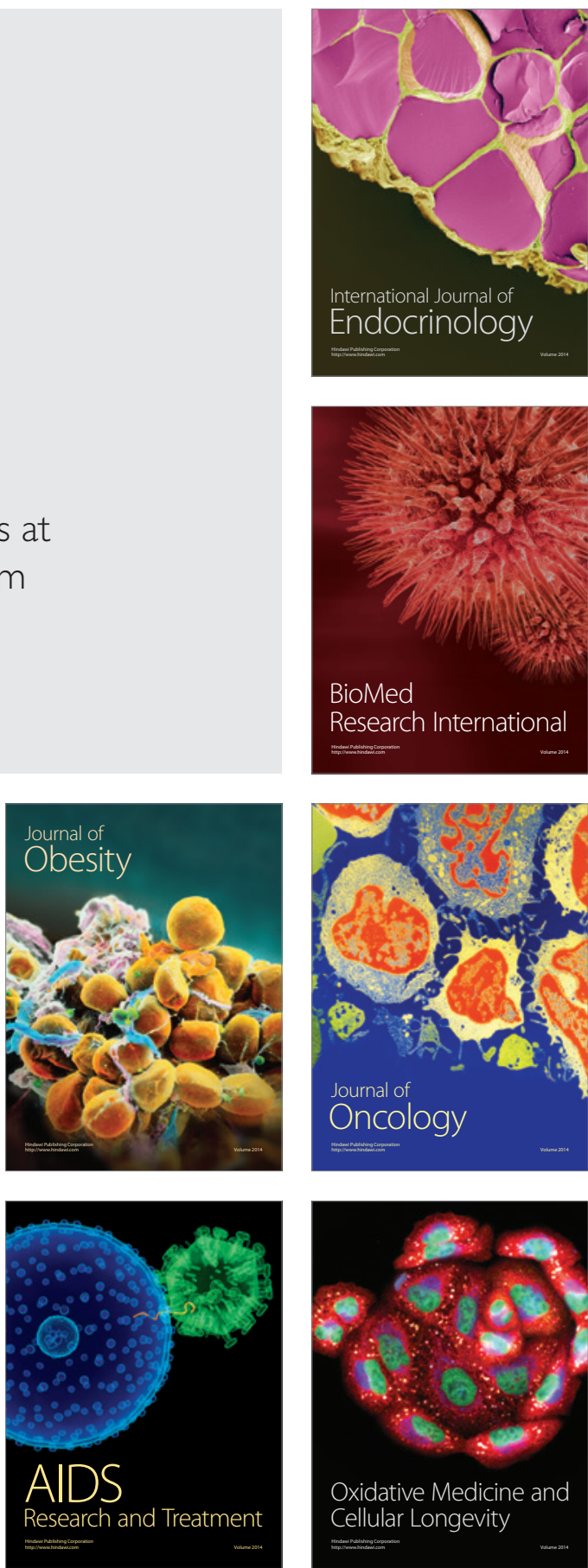